\title{
Guest editorial: special issue on biologically inspired music, sound, art and design
}

\author{
Juan Romero • Penousal Machado • Adrian Carballal
}

Received: 15 April 2013/Published online: 26 April 2013

(C) Springer Science+Business Media New York 2013

\section{Introduction}

The idea of using evolutionary computation for artistic purposes can be traced back to Dawkins. In his 1987 book, The Blind Watchmaker [1], Dawkins presents a program that allows the evolution of the morphology of "virtual creatures". The user guides the genetic algorithm, indicating the favorite biomorphs, avoiding the need to develop a fitness function. Using a similar approach, the seminal works of Sims [2], where genetic programming was used to evolve populations of images, and of Todd and Latham [3], where evolutionary techniques are used to evolve 3D forms with organic appearance, led to the emergence of a new research area, evolutionary art, which is characterized by the use of nature-inspired computing for artistic purposes. At the same time, early works in the field of evolutionary music explored the development of hard-wired functions [4] and of artificial neural networks [5] to assign fitness. Soon afterwards, Baluja et al. [6] applied artificial neural networks to guide the evolution of images.

As is often the case in new research areas, particularly interdisciplinary ones, the first years were characterized by individual efforts and uncoordinated research, which often led to the reinvention of the wheel. This problem was amplified by the lack of scientific events specifically dedicated to the area, which led to the publication of these early efforts in generic evolutionary computation, artificial intelligence, neural networks or computer music conferences and journals.

\footnotetext{
J. Romero $(\bowtie) \cdot$ A. Carballal

Faculty of Computer Science, University of A Coruña, A Coruña, Spain e-mail: jj@udc.es

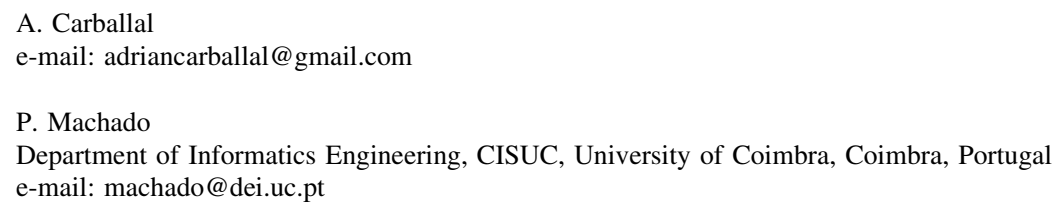


Several researchers made invaluable contributions to overcome this situation. The 1998 Artificial Intelligence in Design Workshop on Evolutionary Design [7], organized by Bentley, was one of the first dedicated venues for dissemination of evolutionary art and design research. It was followed by the 1999 Artificial Intelligence and the Simulation of Behaviour workshop on Creative Evolutionary Systems [8] and by the special issue on Creative Evolutionary Systems [9] of the Applied Intelligence journal, chaired and edited by Bentley and Corne. The books Evolutionary Design by Computers, Peter Bentley (Ed.) [10], and Creative Evolutionary Systems, Bentley and Corne (Eds.) [11], although not entirely dedicated to evolutionary art and music, gave a considerable exposure to the field. The 2000 Genetic and Evolutionary Computation Conference workshop on Genetic Algorithms in Visual Art and Music [12], chaired by Johnson and Romero, was the first event of the field integrated in a major evolutionary computation conference; Two special sections of Leonardo $[13,14]$ were published as follow up to this event, promoting the visibility of the field among art practitioners. In 2001 and 2003 Bilotta et al. chaired the European Conference on Artificial Life workshops on Artificial Life Models for Musical Applications [15, 16]. In 2003 Miranda edited the special issue Evolutionary Music: At the Crossroads of Evolutionary Computing and Musicology [17] of the Contemporary Music Review journal.

In 2003 Johnson and Romero created the Working Group on Music and Art within the European Network of Excellence in Evolutionary Computing (EvoNet). This working group was instrumental for the development of the research community and led to the creation of the Evolutionary Music and Art (evomusart) workshop. The main goal of the event was to promote the growth of the research area by allowing researchers and artists to come together and present their works, while enforcing the same scientific standards as other areas, thus demonstrating that it is possible to develop high-quality scientific research in this creative domain.

With 13 submissions and 11 programme committee members, the first edition of evomusart, chaired by Romero and Johnson, took place in 2003, Essex, UK. The event was one of the Applications of Evolutionary Computing [18] workshops, which were held together with the European Conference on Genetic Programming (EuroGP) and the European Conference on Evolutionary Computation in Combinatorial Optimisation (evoCOP).

The evomusart workshop continued to meet annually. It grew steadily in terms of number of submissions, programme committee members, and quality of submissions, asserting itself as the only yearly event on the field and also as its main forum. The increase in number of submissions can be observed in figure 1. In these 10 years evomusart received a total of 297 paper submissions. On average, $37.7 \%$ of the papers were accepted for oral presentation during the event, while $15.5 \%$ were accepted for poster presentation. In 2012 the program committee was composed of 79 members from 20 different countries and 5 continents.

As a consequence of its success and sustained growth in 2012 evomusart transformed into the International Conference on Evolutionary and Biologically Inspired Music, Sound, Art and Design. Albeit with independent status and proceedings, evomusart is co-located with other conferences focused on specific areas of evolutionary computation, at an annual event now known as evostar. 


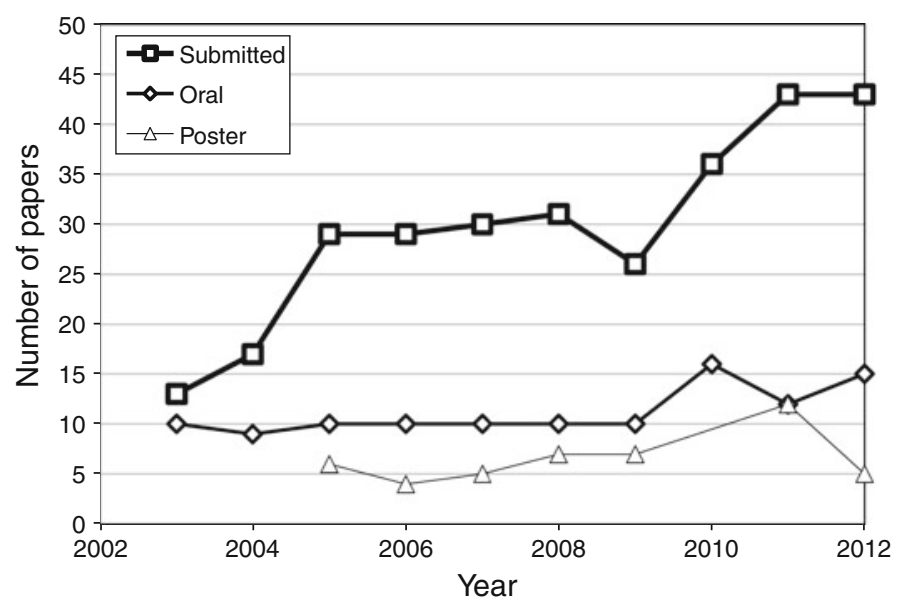

Fig. 1 Number of papers submitted to evomusart, accepted for oral presentation and accepted for poster presentation

In these 10 years the area has also grown significantly. Currently, the main evolutionary computation conferences have tracks, workshops or competitions dedicated to evolutionary art and music or closely related fields. For instance, in 2012, the Genetic and Evolutionary Computation Conference (GECCO) [19] included an Evolutionary Music workshop, a Digital Entertainment Technologies and Arts track and an Evolutionary Art, Design, and Creativity competition, while the IEEE Congress on Evolutionary Computation (CEC) [20] included an Evolutionary Music workshop.

Books such as The Art of Artificial Evolution: A handbook on evolutionary art and music [21] and Evolutionary Computer Music [22] provide a comprehensive view of the area, highlighting hot topics of research and specific challenges, both technically and theoretically. They include thorough survey chapters on visual evolutionary art and design [23] and on evolutionary music [24].

The present special issue celebrates the 10th anniversary of evomusart and its transformation into a conference. The 10th evomusart [25], from which the papers in this special issue were selected, took place 11-13 April, 2012, in Malaga, Spain. The papers that were rated highest by the reviewers and editors were selected and their authors invited to submit an extended version of their work to this special issue. These papers went through an extensive review process, which eventually led to the acceptance of the four papers presented herein.

A significant percentage of works in this area adopts an interactive evolution approach. Byrne et al. propose two new interfaces for user-guided evolution. The paper describes a series of experiments investigating the ability of users to direct search during interactive evolution, as applied to bridge design. The underlying representation is a context-free grammar, and the users have various degrees of direct control over how to apply genetic operators. The results show that users can direct search towards a target design with some success. 
Artistic "value" relates to aesthetics, novelty, style, creativity, culture, context, etc. As such, estimating artistic value or, paraphrasing one of the papers, "judging beauty" is an extremely complex problem, involving areas such as cognitive sciences, psychology, neuroscience, art and music theory, sociology, philosophy, visual perception and audition. The development of automatic fitness assignment schemes has become a hot topic in evolutionary art, which is yearly addressed by several evomusart papers and is the subject of two of the papers of this special issue.

$\mathrm{Li}$ et al. introduce an adaptive model of the user's aesthetic judgements. They explore several image features-color moments, lightness, texture, complexity, order-proposed in previous research and create a relevant subset via feature selection based on information gain. They present a semi-automatic evolutionary art system, which creates a model of the users' aesthetic judgements based on their past choices, predicting their preferences for future generations. The experimental results indicate that this approach may reduce user fatigue. The methodology presented by $\mathrm{Li}$ et al. is particularly relevant for the contextual assessment of the relevance of different image features for evolutionary art purposes.

Bergen and Ross extend existing mathematical models of 2D image aesthetics to the $3 \mathrm{D}$ realm. They present an evolutionary algorithm for the evolution of $3 \mathrm{D}$ structures, which uses a multi-objective fitness function constructed using features based on entropy, complexity, deviation from normality, 1/f noise, and symmetry. A user study demonstrates preference for the evolved forms over randomly generated ones.

Representation is key to evolutionary computation in general and to evolutionary art in particular. McDermott introduces graph grammars as a representation for evolutionary $3 \mathrm{D}$ design, comparing the results obtained using graph grammars and a direct graph representation. He defines a set of low-level features measuring qualities of the graphs' size and shape, connectivity, and complexity. Using these features, linear classifiers can be trained to separate the designs resulting from the two representations, and to separate good from bad designs as judged by users. The use of graph grammars for the evolution of 3D designs is illustrated by runs using interactive evolution and automatic evolution with arbitrary target feature vectors.

Continuing the tradition represented in this special issue, evomusart 2013 has recently been held in Vienna, Austria. We look forward to evomusart 2014, which will be held in Baeza, Spain, and we take this opportunity to encourage readers to attend and to submit their best work.

The guest editors thank all the authors who submitted their work to evomusart, and all the reviewers who dedicated their time and scientific expertise to ensure the high quality of the papers presented at the conference and in this special issue. We express our gratitude towards Colin G. Johnson, one of the founders of evomusart and chair in 2003 and 2004, Jon McCormack, chair in 2008 and 2009 and Gary Greenfield, chair in 2010 and 2011, for their enthusiasm, support and hard work. We would also like to acknowledge the organizers of the art exhibitions associated with evomusart - Paulo Urbano, Anna Esparcia, Tim Blackwell and Janis Jefferies - and the local chairs who created the conditions for these exhibitions to take place, Anikó Ekárt and Anna Esparcia. 
A heartfelt thanks goes to Jennifer Willies from Edinburgh Napier University. Ever since its inaugural meeting in 2003 evomusart has relied on her dedicated work and continued involvement. Without her, evomusart could not have achieved its current status. Finally, we would like to thank Lee Spector for his openness, help and encouragement to this project, and Geetha Subramanian of Springer for all her helpfulness during this process.

\section{References}

1. R. Dawkins, The Blind Watchmaker: Why the Evidence of Evolution Reveals a Universe Without Design. (W.W. Norton and Company, Inc., New York, 1987)

2. K. Sims, Artificial evolution for computer graphics. ACM Comput. Graph. 25, 319-328 (1991)

3. S. Todd, W. Latham, Evolutionary Art and Computers. (Academic Press, Winchester UK, 1992)

4. A. Horner, D. Goldberg, Genetic algorithms and computer-assisted composition. In: Genetic Algorithms and Their Applications: Proceedings of the Fourth International Conference on Genetic Algorithms, (1991). pp. 427-441

5. P.M. Gibson, J.A. Byrne, Neurogen, musical composition using genetic algorithms and cooperating neural networks. In: Artificial Neural Networks, 1991., Second International Conference on, (1991). pp. 309-313

6. S. Baluja, D. Pomerlau, J. Todd, Towards automated artificial evolution for computer-generated images. Connect. Sci. 6(2), 325-354 (1994)

7. P. Bentley, A summary of evolutionary design. In Artificial Intelligence in Design, Workshop on Evolutionary Design, ed. by P. Bentley (Lisbon, Portugal 1998)

8. P. Bentley, D. Corne, (eds.), Proceedings of the AISB'99 Symposium on Creative Evolutionary Systems. Society for the Study of Artificial Intelligence and Simulation of Behaviour, (Endinburgh, 1999)

9. P. Bentley, D. Corne, Guest editorial for special issue on creative evolutionary systems. Appl. Intell. 16(2), 99-100 (2002)

10. P. Bentley, (ed.), Evolutionary Design by Computers (Morgan Kaufman, San Francisco, California, USA, 1999)

11. P. Bentley, D. Corne, (eds.), Creative Evolutionary Systems (Morgan Kaufman, San Francisco, California, USA, 2001)

12. C.G. Johnson, J.J.R. Cardalda, (eds.), Workshop on Genetic Algorithms in Visual Art and Music, Proceedings of the Genetic and Evolutionary Computation Conference (Morgan Kaufmann, Las Vegas, Nevada, USA, 2000)

13. C. Johnson, J. Romero, Genetic algorithms in visual art and music. Leonardo 35(2), 175-184 (2002)

14. C. Johnson, J. Romero, Genetic algorithms in visual art and music. Leonardo 36(1), 43-64 (2003)

15. J. Kelemen, P. Sosík, (eds.), Advances in Artificial Life, 6th European Conference, ECAL 2001, Prague, Czech Republic, September 10-14, 2001, Proceedings, Lecture Notes in Computer Science, vol. 2159, (Springer, 2001)

16. W. Banzhaf, T. Christaller, P. Dittrich, J.T. Kim, J. Ziegler, (eds.), Advances in Artificial Life, 7th European Conference, ECAL 2003, Dortmund, Germany, September 14-17, 2003, Proceedings, Lecture Notes in Computer Science, vol. 2801, (Springer 2003)

17. E.R. Miranda, Evolutionary music: at the crossroads of evolutionary computing and musicology. Contemp. Music Rev. 22(3), 115 (2003)

18. G.R. Raidl, J.A. Meyer, M. Middendorf, S. Cagnoni, J.J.R. Cardalda, D. Corne, J. Gottlieb, A. Guillot, E. Hart, C.G. Johnson, E. Marchiori, (eds.), Applications of Evolutionary Computing, EvoWorkshop 2003: EvoBIO, EvoCOP, EvoIASP, EvoMUSART, EvoROB, and EvoSTIM, Essex, UK, April 14-16, 2003, Proceedings, Lecture Notes in Computer Science, vol. 2611, (Springer 2003)

19. T. Soule, J.H. Moore, (eds.), Genetic and Evolutionary Computation Conference, GECCO '12, ACM, (Philadelphia, PA, USA, 2012)

20. H. Abbass, D. Essam, R. Sarker, (eds.), Proceedings of the IEEE Congress on Evolutionary Computation, CEC, IEEE (Brisbane, Australia, 2012) 
21. J. Romero, P. Machado, (eds.), The Art of Artificial Evolution: A Handbook on Evolutionary Art and Music. Natural Computing Series (Springer, Berlin Heidelberg 2007). doi:10.1007/978-3-540-72877-1

22. E.R. Miranda, J.A. Biles, Evolutionary Computer Music. (Springer, Secaucus, NJ, 2007)

23. M. Lewis, Evolutionary visual art and design. In The Art of Artificial Evolution: A Handbook on Evolutionary Art and Music, ed. by J. Romero, P. Machado. (Springer, Berlin Heidelberg, 2007), pp. 3-37. doi:10.1007/978-3-540-72877-1_1

24. J.A. Biles, Evolutionary computation for musical tasks. In Evolutionary Computer Music, ed. by E.R Miranda, J.A. Biles. (Springer, London, 2007), pp. 28-51. doi:10.1007/978-1-84628-600-1_2

25. P. Machado, J. Romero, A. Carballal, (eds.), Evolutionary and Biologically Inspired Music, Sound, Art and Design-First International Conference, EvoMUSART 2012, Málaga, Spain, April 11-13, 2012. Proceedings, Lecture Notes in Computer Science, vol. 7247, (Springer, 2012) 